\title{
Particle Identification with the LHCb RICH System
}

\author{
Neville Harnew ${ }^{1}$ \\ Denys Wilkinson Building, University of Oxford, Oxford OX1 3RH, UK.
}

\begin{abstract}
The LHCb experiment uses a Ring Imaging Cherenkov (RICH) system to provide particle identification over the momentum range 2-100 GeV/c. Two RICH detectors are employed. The upstream detector, RICH 1, utilizes both aerogel and $\mathrm{C}_{4} \mathrm{~F}_{10}$ gas radiators whilst the downstream RICH 2 uses a $\mathrm{CF}_{4}$ gas radiator. The RICH 2 detector has been fabricated and is installed in the LHCb interaction region; RICH 1 has a programme of phased design and construction. Novel Hybrid Photon Detectors (HPDs) have been developed in collaboration with industry to detect the Cherenkov photons in the wavelength range 200-600 $\mathrm{nm}$. The HPDs are enclosed in iron shielding and Mumetal cylinders to allow operation in magnetic fields up to $50 \mathrm{mT}$. The performance of pre-series HPDs and the results obtained from a particle test beam using the full $\mathrm{LHCb}$ readout chain is presented. The production of a total of 484 HPDs required for the two RICH detectors has recently commenced. The expected performance of the LHCb RICH system, obtained from realistic simulation, is described.
\end{abstract}

Key words: LHCb experiment, RICH detectors, Hybrid Photon Detectors, Aerogel, Beryllium mirrors PACS:

\section{Introduction}

The LHCb experiment [1] will make precision measurements of $\mathrm{CP}$ violation and rare decays of $\mathrm{B}$ hadrons at the Large Hadron Collider (LHC). The LHCb detector, shown in Fig. 1, is a forward spectrometer. This design is optimized to accept the decay products of $b \bar{b}$-quarks which are produced with a strong angular correlation in the forwardbackward directions.

Particle identification is essential to separate pions from kaons in selected B meson decays. Two

\footnotetext{
1 On behalf of the LHCb RICH Collaboration
}

Ring Imaging Cherenkov (RICH) detectors perform $\pi / \mathrm{K}$ separation from 2 to $\sim 100 \mathrm{GeV} / \mathrm{c}$ [2]. The polar angular acceptance, in the spectrometer bending plane, of the upstream RICH 1 is from 25 to $300 \mathrm{mrad}$, while that for the downstream RICH 2 is $15-120 \mathrm{mrad}$. The properties of the $\mathrm{RICH}$ detectors are summarized in Table 1.

Position-sensitive photon detectors are required to detect and reconstruct the Cherenkov rings. Pixel Hybrid Photon Detectors (HPDs) have been adopted [3]. A total of 484 HPDs cover the $\sim 2.6 \mathrm{~m}^{2}$ total photon detection area, consisting of 196 HPDs in RICH 1 and 288 in RICH 2. 


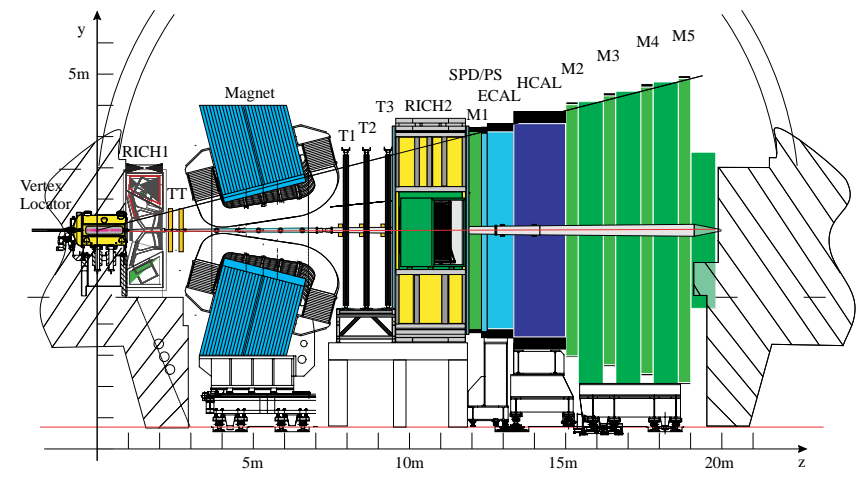

Fig. 1. A schematic of the $\mathrm{LHCb}$ detector.

\begin{tabular}{|c|c|c|c|}
\hline \multirow[b]{2}{*}{ Property / Radiator } & \multicolumn{2}{|c|}{ RICH 1} & \multirow{2}{*}{$\begin{array}{c}\mathrm{RICH} 2 \\
\mathrm{CF}_{4}\end{array}$} \\
\hline & Aerogel & $\mathrm{C}_{4} \mathrm{~F}_{10}$ & \\
\hline Radiator length (mm) & 50 & 850 & 1960 \\
\hline Refractive index & 1.03 & 1.0014 & 1.0005 \\
\hline Photo-electrons/track & 6.8 & 31 & 23 \\
\hline Angular resolution (mrad) & 2.6 & 1.6 & 0.7 \\
\hline Momentum range $(\mathrm{GeV} / \mathrm{c})$ & $\sim 2-10$ & $<65$ & $<100$ \\
\hline
\end{tabular}

\section{The RICH 1 detector}

A schematic of the RICH 1 detector is shown in Fig. 2. The detector is split vertically, with two sets of spherical and plane mirrors focusing the Cherenkov photons onto two photon detector arrays. Two radiator materials are employed, aerogel and $\mathrm{C}_{4} \mathrm{~F}_{10}$ gas.

RICH 1 employs solid silica aerogel for the identification of low momentum particles from 2 to $10 \mathrm{GeV} /$ c. A substantial R\&D effort has been employed in the aerogel manufacturing process, resulting in aerogel of unprecedented optical quality and tile size [4]. The transparency of aerogel, $T$, is parameterised by the expression:

$$
T=A \cdot e^{-C \frac{t}{\lambda^{4}}}
$$

where $t$ is the thickness of the aerogel in $\mathrm{cm}, \lambda$ is the photon wavelength in $\mu \mathrm{m}, C$ is the clarity coeffi-

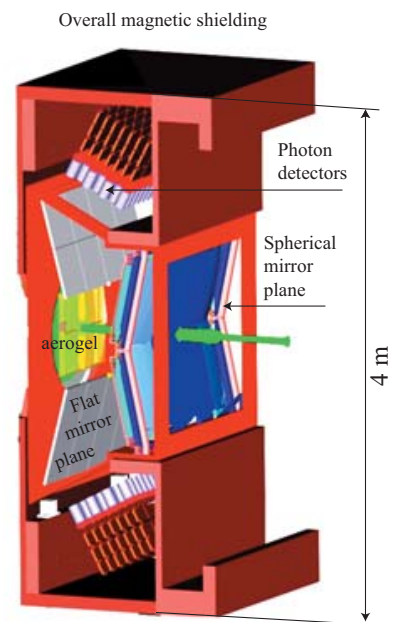

RICH 1

Fig. 2. A schematic of the RICH 1 detector.

cient in units of $\mu \mathrm{m}^{4} / \mathrm{cm}$ and $A$ is the transparency as $\lambda \rightarrow \infty$. Large aerogel tiles, $20.0 \times 20.0 \times 5.1 \mathrm{~cm}^{3}$, have been tested for use in RICH 1 . The aerogel has a nominal refractive index of 1.030 at $400 \mathrm{~nm}$ and a clarity of $0.0060 \mu \mathrm{m}^{4} / \mathrm{cm}$. The aerogel is stable towards irradiation and shows no change in transparency when tested after an accumulated fluence of up to $5.5 \cdot 10^{13} / \mathrm{cm}^{2}$ of neutrons or protons, or a $\gamma$ dose of $\sim 2.5 \cdot 10^{5} \mathrm{~Gy}$.

Beryllium spherical mirrors of $4 \mathrm{~mm}$ thickness with radius of curvature of $2700 \mathrm{~mm}$ will focus the Cherenkov light. The very low atomic number of beryllium ensures a low fraction of a radiation length, $\sim 1 \%$ of $\mathrm{X}_{0}$, which is essential to minimize secondary interactions in the spectrometer acceptance. The first of the eight spherical mirrors has recently been delivered. The radius of curvature is measured to be within $1 \%$ of nominal, and the point image diameter $\left(d_{0}\right)$ at the mirror centre of curvature is $3.3 \mathrm{~mm}$, close to the nominal $2.5 \mathrm{~mm}$. The remaining seven mirrors will be delivered by the end of 2006.

It is crucial to shield the photon detectors of RICH 1 from stray fields of the LHCb dipole magnet. Iron magnetic shielding, shown in Fig. 2, also funnels the field such that the field integral between the $\mathrm{LHCb}$ vertex detector and the first tracking station is high enough to be useful for the trigger [1]. The shield has recently been fabricated and in- 
stalled in its nominal position. Measurements in the field of the $\mathrm{LHCb}$ magnet have confirmed a maximum value of $25 \mathrm{mT}$ in the region where the photon detectors will be placed.

The RICH 1 detector is currently undergoing a phased programme of design and construction, and will be commissioned at the end of 2006 .

\section{The RICH 2 detector}

A schematic of the RICH 2 detector is shown in Fig. 3. The detector is split horizontally, with two sets of spherical and plane mirrors focusing the Cherenkov photons onto two photon detector arrays.

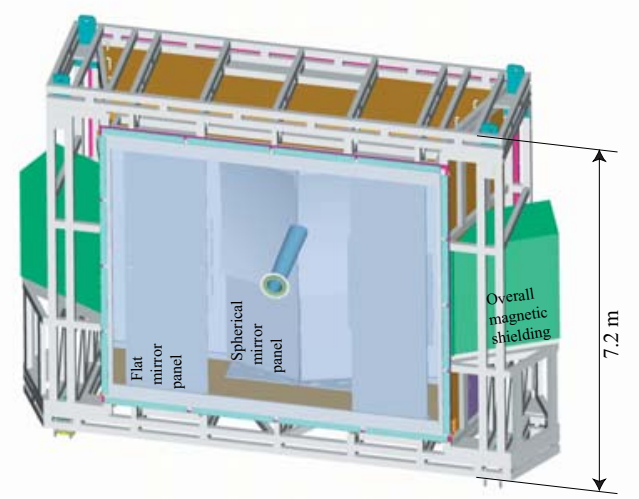

Fig. 3. A schematic of the RICH 2 detector.

RICH 2 contains 56 spherical mirrors made from $6 \mathrm{~mm}$ thin glass substrates and 40 flat mirrors. Since the detector is located after the tracking system and immediately upstream of the calorimeter system, a higher material budget for the optical system can be tolerated. The alignment of the optical system for RICH 2 was achieved in three stages [5]. Firstly each spherical mirror was aligned in turn by shining a laser from the mirror centre of curvature, and the mirror adjusted until the laser reflected back to the common point, measured with a CCD camera. Then a sample of flat mirrors, paired with their respective spherical elements, were aligned to their theoretical centres. Finally the remainder of the flat mirrors were aligned with respect to these. The estimated contribution from the alignment and stability of the optical system to the overall uncertainty of the single photon resolution was determined to be of order $\sim 150 \mu \mathrm{rad}$, with a contribution of $\sim 50 \mu \mathrm{rad}$ for the spherical mirrors alone.

The RICH 2 superstructure has been assembled with a carbon fibre-polymeric foam entrance window and aluminium-skin, foam-core, exit window. Magnetic shielding for the photon detectors has been mounted on each side of the superstructure, and the magnetic field mapped inside the structure. The RICH 2 detector was transported to its final position inside the experimental cavern in November 2005.

\section{The Photon Detectors}

The Hybrid Photon Detector, shown in Fig. 4, has been developed in collaboration with industry to detect the Cherenkov photons in the wavelength range 200-600 $\mathrm{nm}$ [3]. The HPD consists of a vacuum tube with a $7 \mathrm{~mm}$ thick quartz entry window, giving a sensitive diameter of $72 \mathrm{~mm}$. An S20 photo-cathode is deposited on the inner surface of the window. The photo-electrons are accelerated by a cross-focusing electrostatic field onto an anode, using a tetrode electron optics and a demagnification factor of five. The anode is a pixel silicon sensor to which is bump-bonded a LHCBPIX1 readout chip [6], operating at the LHC frequency of $40 \mathrm{MHz}$. At the operating voltage of $20 \mathrm{kV}$, the photo-electrons are accelerated to produce about 5000 electron-hole pairs in the silicon sensor. A total of $32 \times 32$ channels per HPD are read out, giving an effective granularity of $2.5 \times 2.5 \mathrm{~mm}^{2}$ at the photo-cathode. The HPDs are mounted in a closepacked arrangement, with an active area fraction of $\sim 65 \%$. The RICH detectors will be equipped with 484 HPDs in total.

The production of the HPDs is a logistic challenge involving seven companies and production sites. The most crucial steps are the bump-bonding of the silicon detector to the readout chip using a high temperature solder, the packaging of this assembly into the ceramic carrier, and the encapsula- 


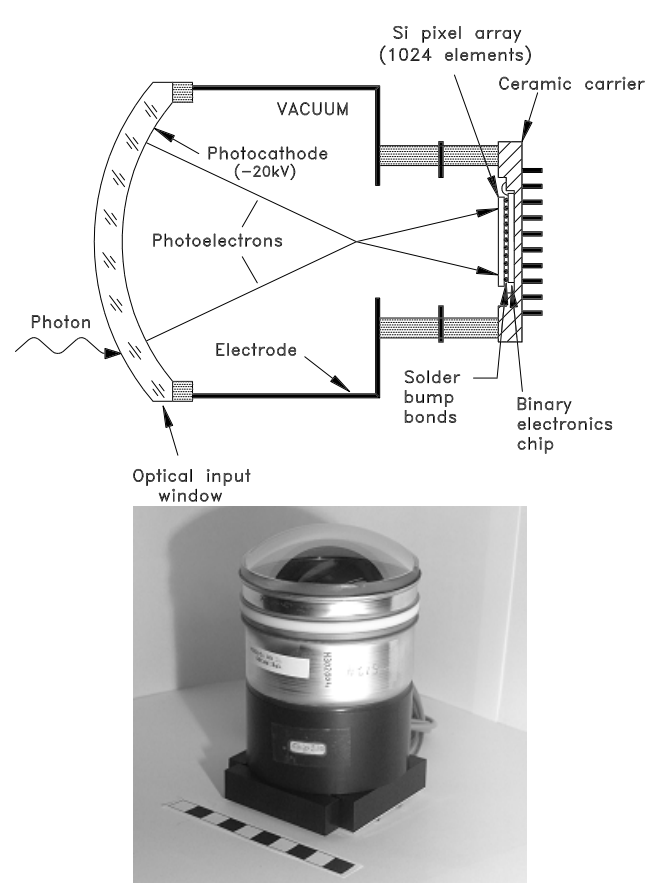

Fig. 4. Schematic of a Hybrid Photon Detector and photograph with a $10 \mathrm{~cm}$ scale.

tion of the the carrier into the photo-tube and deposition of the photo-cathode. The HPDs are then subject to a series of tests to provide quality assurance before they are accepted for use in LHCb.

The results for nine pre-series HPDs, compared to specifications, are summarised in Table 2. Apart from a few exceptions, the performance matches or exceeds the requirements. Even in those cases where the specifications are missed, the HPDs are still suitable for use in LHCb. The quantum efficiencies (QEs) of the photo-cathodes of the nine pre-series HPDs are shown in Fig. 5. Typical QEs are $\sim 25 \%$ at $270 \mathrm{~nm}$; the specification points are given as crosses in the figure. The shapes of the curves are defined by the cut-off of the quartz window at $200 \mathrm{~nm}$ and the properties of the S20 photocathode leading to the variations in the near UV and the visible region. For eight of the HPDs, the specifications are well exceeded.

Pixel HPDs have been subject to stringent aging tests. An HPD has been exposed to intense LED photo-cathode illumination at $40 \%$ occupancy at $50 \mathrm{C}$ for 1 month, equivalent to 10 years of $\mathrm{LHCb}$ running (the normal occupancy is $1 \%$ ). No degra-

\begin{tabular}{lll}
\hline Item & Spec. & Typ. results \\
\hline Threshold & $<2000 e^{-}<1200 e^{-}$ \\
Noise & $<250 e^{-}$ & $<160 e^{-}$ \\
Pixel response & $>95 \%$ & $>99 \%$ \\
Ion feedback rate rel. to signal & $<10^{-2}$ & $<10^{-3}$ \\
Dark count rate $\left[\mathrm{kHz} / \mathrm{cm}^{2}\right]$ & $<5$ & $0.03-3.0$ \\
Typ. leakage current $@ 80 \mathrm{~V}$ bias $1 \mu \mathrm{A}$ & $<1 \mu \mathrm{A}$ \\
PE det. eff. & typ. $85 \%$ & $79-89 \%$ \\
\hline Table 2 & &
\end{tabular}

The main specifications of the HPDs together with the typical results obtained from the first nine pre-series tubes.

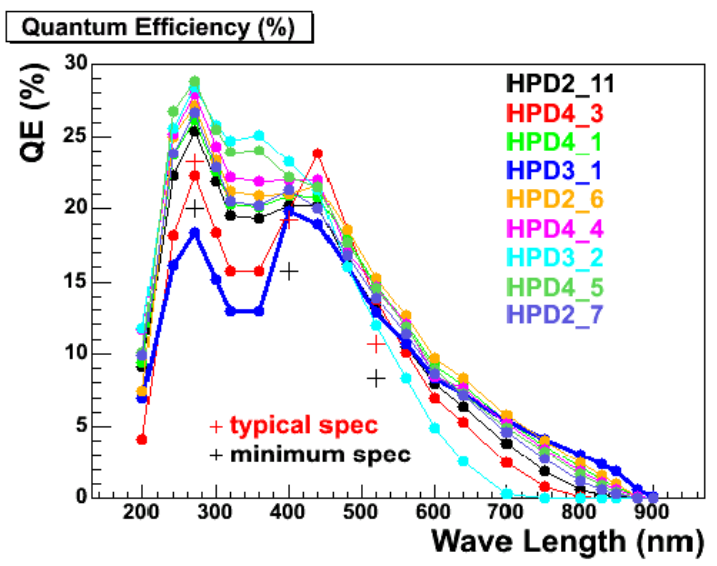

Fig. 5. The measured quantum efficiencies of nine pre-series HPDs.

dation has been observed of QE, dark current, ion feedback rate or light yield [7]. The ion feedback rate, resulting from collisions of accelerated electrons with residual gas ions, increased from 1 to $3 \%$ over the exposure period but recovered afterwards.

The HPDs must operate in a field of up to $25 \mathrm{mT}$ in the RICH 1 region. Each HPD can be shielded effectively with an individual mu-metal cylinder placed around it [8]. A correction must be made for an electron image rotation due to the $\mathrm{E} \times \mathrm{B}$ effects within the tube and angular parameterisations are made for both axial and transverse field distortions [9]. Studies show that for a shielded HPD in $30 \mathrm{mT}$, there is no loss of active HPD area; at $50 \mathrm{mT}$ the image loss would be $\sim 5 \%$. 


\section{System tests}

Beam tests were performed with a prototype RICH detector at the CERN T9 facility using $10 \mathrm{GeV} / \mathrm{c}$ negative pions and electrons. Beam particles entered the vessel through a thin aluminum foil and passed through $\sim 1 \mathrm{~m}$ length of $\mathrm{N}_{2}$ radiator gas. Cherenkov light was focused by a parabolic mirror onto a photo-detector plane containing six pre-series HPDs. Saturated $(\beta=1)$ electron rings were fully contained within a single HPD, and each HPD was sampled in turn. The HPDs operated at the LHC frequency of $40 \mathrm{MHz}$, read out via a full prototype electronics readout chain [10].

A ring image obtained is shown in Fig. 6, integrated over $\sim 30 \mathrm{k}$ events. Very few hits are seen outside of the ring, indicating a low level of noise. The distribution of the number of measured photoelectrons (PEs) per event was fitted with a Poisson distribution, modified for known inefficiencies. The data and the fit are shown for a typical HPD in Fig. 7.

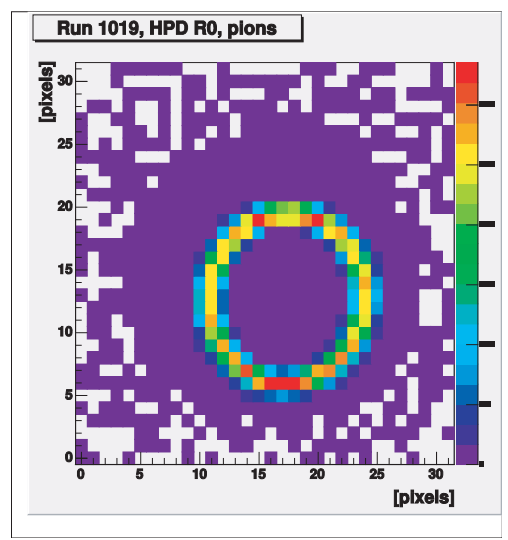

Fig. 6. Ring image on an HPD from a $10 \mathrm{GeV} / \mathrm{c}$ pion beam and $\mathrm{N}_{2}$ radiator, integrated over $\sim 30 \mathrm{k}$ events.

A summary of the performance for all HPDs is given in Table 3 . The uncertainty in the expectation is dominated by measurements of the quantum efficiency of the HPDs and is at the $10 \%$ level. The data are generally in good agreement with the expectations. The discrepancy for HPD 1 originates from a known timing problem. The good overall performance meets the $\mathrm{LHCb}$ requirements.

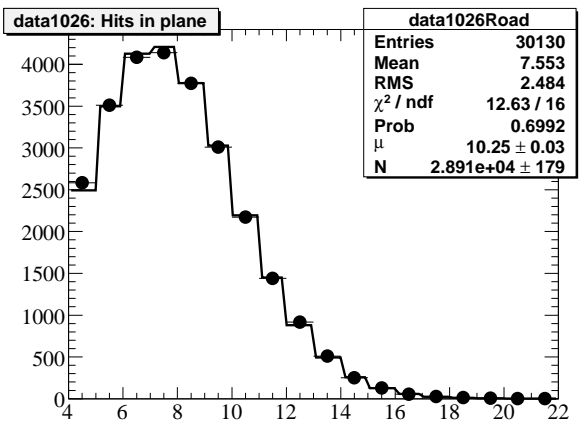

Fig. 7. The number of PEs observed on a single HPD (points). The Poisson fit to these data is also shown (solid line).

HPD $\mu$ (measured) $\mu$ (expected) Ratio

\begin{tabular}{llll}
\hline 1 & 7.78 & 6.02 & 1.29 \\
2 & 11.5 & 11.2 & 1.03 \\
3 & 8.8 & 8.9 & 0.99 \\
4 & 9.7 & 10.7 & 0.90 \\
5 & 10.1 & 10.0 & 1.01 \\
6 & 8.5 & 11.4 & 0.75 \\
\hline
\end{tabular}

Table 3

The measured mean photo-electron yields compared with expectations for the six prototype HPDs.

\section{RICH performance studies}

The LHCb RICH performance has been extensively simulated in the GEANT4 Monte Carlo framework including realistic tracking and effects of all known background processes. A global pattern recognition and ring-fitting approach has been adopted, with simultaneous assignment of rings to tracks in RICH 1 and RICH 2 [11]. A probability distribution is determined for finding photons in each pixel of the detector plane for a given set of track identification hypotheses. This is compared with the observed hit distribution to determine a likelihood; the particle identification hypotheses are then adjusted to maximize the likelihood. Distributions of hit HPD pixels on the HPD planes in RICH 1 and RICH 2 are shown for a typical B event in Fig. 8. Rings characteristic of 

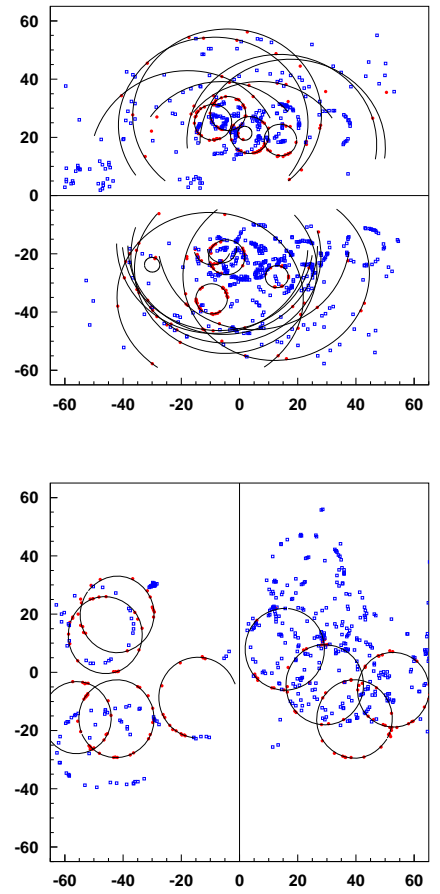

Fig. 8. Distributions of hit HPD pixels on the HPD planes of RICH 1 (top) and RICH 2 (bottom). Units are in $\mathrm{cm}$.

the three radiators can be clearly seen. The global fits to the Cherenkov rings which have associated tracks are superimposed.

The expected RICH performance from the Monte Carlo simulation is shown in Fig. 9, including all known background processes. The figures show the efficiency for pions identified as "light" particles $(\mathrm{e}, \mu, \pi)$, and kaons identified as "heavy" particles $(\mathrm{K}, \mathrm{p})$ for those tracks which completely pass through the LHCb spectrometer. Efficiencies typically above $90 \%$ are observed for tracks between $20-80 \mathrm{GeV} / \mathrm{c}$. The probabilities of particle misidentification are typically below $10 \%$ over the same momentum range.

The effect of the $\mathrm{RICH}$ detectors in reducing background in the channel $\mathrm{B} \rightarrow \pi^{+} \pi^{-}$is shown in Fig. 10. Without the RICH information, the signal is swamped by other $B_{d}$ and $B_{s}$ decay modes. After applying the RICH identification, the background from competing channels is reduced below the $10 \%$ level.
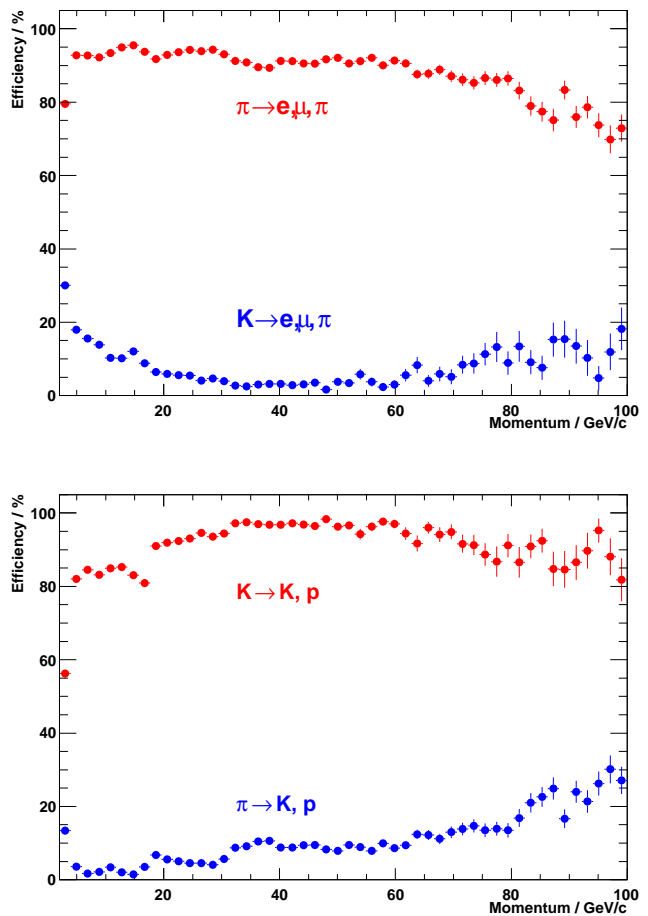

Fig. 9. The efficiencies and misidentification probabilities (in \%) as a function of momentum for pions identified as "light" (top) and kaons identified as "heavy" (bottom).

\section{Summary and conclusions}

The LHCb experiment uses two RICH detectors to provide particle identification over the momentum range $2-100 \mathrm{GeV} / \mathrm{c}$. RICH 1 is currently undergoing a phased programme of design and construction. RICH 2 has been fabricated, aligned and installed in the $\mathrm{LHCb}$ experimental area. The performance of the Hybrid Photon Detectors has been successfully characterized in laboratory and beam tests. Cherenkov light yields and resolutions agree with expectations. The HPDs are currently being produced in industry at the rate of $\sim 30$ per month for completion in February 2007. The LHCb RICH system will be ready for first LHC collisions in 2007.

\section{Acknowledgments}

I would like to thank colleagues in the $\mathrm{LHCb}$ 

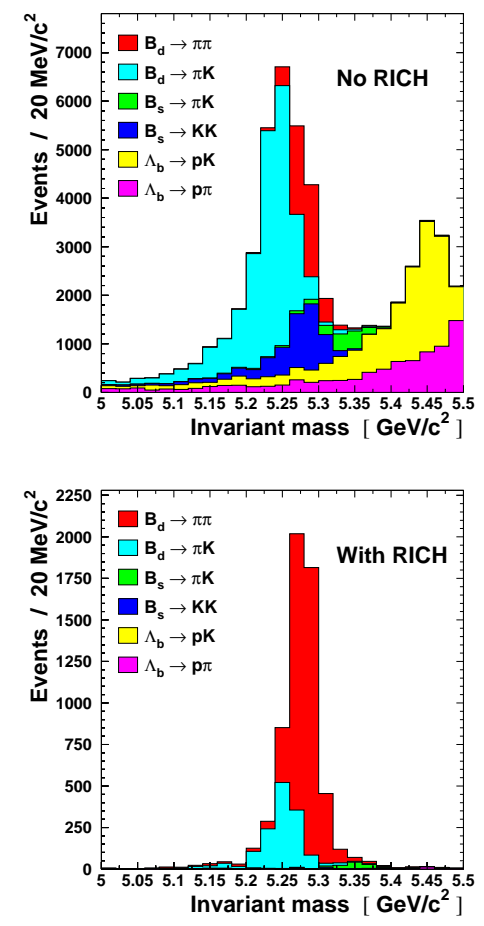

Fig. 10. The effect of the RICH in the selection of the channel B $\rightarrow \pi^{+} \pi^{-}$without (top) and with (bottom) using RICH information.

Collaboration who have provided invaluable assistance, in particular S.Eisenhardt, R. Forty, A. Papanestis, M. Patel, O. Ullaland and G. Wilkinson. I also thank the TRD2005 local committee, in particular F. Loparco and P. Spinelli, for making the conference in Ostuni so enjoyable.

\section{References}

[1] The LHCb Collaboration, LHCb Re-Optimized Detector Design and Performance Technical Design Report, CERN/LHCC 2003-030, LHCb TDR9, September 2003.

[2] The LHCb Collaboration, LHCb RICH Technical Design Report, CERN/LHCC 2000-037, LHCb TDR3, 7 September 2000.

[3] M. Campbell et al., Performance of hybrid photon detector prototypes with encapsulated silicon pixel detector and readout for the RICH counters of $\mathrm{LHCb}$, Nucl. Instr. and Meth. A504 (2003), 286.

[4] C. Matteuzzi, A RICH with aerogel for a hadron collider, Nucl. Instr. and Meth. A553 (2005) 356.
[5] C. D'Ambrosio, The RICH 2 Detector of the $\mathrm{LHCb}$ Experiment, submitted to IEEE NSS 2005.

[6] K. Wyllie et al., Silicon detetors and electronics for pixel hybrid photon detectors, Nucl. Instr. and Meth. A530 (2004) 82.

[7] N. Kanaya et al., Performance study of hybrid photon detectors for the LHCb RICH, Nucl. Instr. and Meth. A553 (2005) 41 .

[8] M. Patel et al., Magnetic shielding studies of the $\mathrm{LHCb}$ RICH photon detectors, Nucl. Instr. and Meth. A553 (2005) 114.

[9] G. Aglieri Rinella et al., Characterisation and compensation of magnetic distortions for the pixel Hybrid Photon Detectors of the LHCb RICH, Nucl. Instr. and Meth. A553 (2005) 120.

[10] M. Adinolfi, System test of a three-column $\mathrm{LHCb}$ RICH-2 prototype detector, Nucl. Instr. and Meth. A553 (2005) 328.

[11] R. Forty, RICH pattern recognition for $\mathrm{LHCb}$, Nucl. Instr. and Meth. A433 (1999) 257. 